\title{
ROLE OF KINESIOTHERAPY IN THE RECOVERY OF PATIENTS WITH PRIMARY COXARTHROSIS
}

\author{
POP, Irina Alexandra ${ }^{1}$, DOGARU, Bombonica Gabriela ${ }^{2}$ \\ 1"Iuliu Hatieganu" University of Medicine and Pharmacy Cluj-Napoca \\ ${ }^{2}$ Clinical Rehabilitation Hospital Cluj-Napoca, Romania
}

\begin{abstract}
:
Primary coxarthrosis is a disorder of the hip that may lead to a decrease in the quality of life due to the presence of pain, vicious positions, joint stiffness, and decrease in the joint range of motion. In this article, the role and importance of kinesiotherapeutic treatment along with drug therapy in the recovery of patients with primary coxarthrosis was studied. Kinesiotherapy administered to patients in the study group (medication and kinesiotherapy) contributed to the increase of the joint angle values at the level of the coxofemoral joint and muscle strength. The Womac test performed at the end of treatment (day 12) in the study group shows a decrease in pain intensity and a reduction of joint stiffness compared to day 1 , before the initiation of treatment. The Lequesne functional index applied at the end of the 12 days of treatment from statistical analysis shows that patients can easily walk longer distances and can perform daily activities more easily, which results in an increase in their quality of life.
\end{abstract}

Key words: primary coxarthrosis, kinesiotherapy, recovery.

\section{Introduction:}

Coxarthrosis is a disorder of the hip that results from an imbalance between stress mechanisms exerted on the hip and the exercise resistance capacity of cartilage and bone tissue. Two groups of coxarthrosis are distinguished: primary coxarthrosis, also termed idiopathic, and secondary coxarthrosis. Studies on the etiology of coxarthrosis have shown a frequency of $40 \%$ for primary coxarthrosis and $60 \%$ for secondary coxarthrosis [1].

When no cause can be established at the origin of the syndrome, coxarthrosis is considered to be "primary" or "essential". This type of coxarthrosis is caused by general factors that are little known, which alter the metabolism of joint cartilage and induce premature senescence lesions, through the early wear of joints subjected to maximal strain. In all disease forms, the presence of a joint imbalance is admitted, which depends on the intervention of several risk factors: heredity, nutrition, hormonal status, bone density, cartilage and bone metabolism, obesity, the mechanical joint environment, cartilage loading, joint trauma and deformation, professional strain, sports activities, muscle weakening [2].

Progressive degenerative changes in the hyaline cartilage remain asymptomatic for a long time period. The major symptoms with variable functional implications are pain, which initially occurs after marked mechanical strain, prolonged monotonous standing positions, and which progressively accompanies the patient during the day, and joint stiffness with progressive joint limitation, with a tendency to develop vicious positions (hip flexum in external rotation) [1]. 
From a clinical-functional point of view, three stages are described: initial, middle, and final.

If diagnosis in the period of active disease generally poses no problem, the early diagnosis of the disease that is based on anamnesis, frequently unspecified and inaccurate, as well as on radiographic findings, generates discussions and uncertainties, particularly in its essential forms.

The objectives of treatment in coxarthrosis are the reduction of pain and inflammation, the increase of joint mobility and the maintenance of function, the prevention of physical disability, the avoidance of drug toxicity, the improvement of the quality of life [3].

Treatment involves several aspects: removal of favoring factors, drug therapy, physiotherapeutic treatment and surgical treatment.

The kinesiotherapy program in primary coxarthrosis is aimed at meeting all the four objectives of the treatment of this disease, i.e. the reduction of pain, the increase of mobility and stability, the improvement of coordination and balance during walking.

The evolution of coxarthrosis is slow and progressive. As a rule, it is difficult to evaluate the onset, which is frequently insidious, sometimes marked by acute episodes followed by remission for months or years [4].

\section{Objectives:}

The objectives of the study are the demonstration of the essential role of kinesiotherapy in patients with primary coxarthrosis, which contributes to the prevention of the vicious positions of the hip, the recovery of the function of the coxofemoral joint by kinesiotherapy, the increase of joint mobility, the increase of muscle strength, the improvement and fighting of pain.

\section{Material and method:}

We applied the case study to a study group consisting of 20 patients with primary coxarthrosis from the "Sfântul Iosif" Center of Physiotherapy and Kinesiotherapy in Cluj-Napoca, Romania, in the period April-June 2013. The subjects received drug treatment (anti-inflammatory drugs) as well as kinesiotherapeutic treatment.

In order for the study to be as relevant as possible, we included a control group with a comparable level to that of the study group - a number of 10 patients with primary coxarthrosis, with drug treatment (anti-inflammatory drugs), patients of various family doctors.

The inclusion criterion for patients was the diagnosis of primary coxarthrosis, and the exclusion criterion was represented by patients who had physiotherapy, massage and kinesiotherapy contraindications.

We administered to both groups a patient evaluation record that included: a questionnaire regarding sex, age and the environment of origin, articular testing, muscular testing of the coxofemoral joint, Womac scale for the assessment of pain, stiffness and reduction of joint mobility, Lequesne functional index.

For statistical analysis, we used the technique of parallel groups, using two subject groups (study group - patients with drug treatment and kinesiotherapeutic treatment, and control group - patients with drug treatment).

In this intersubject study design, we comparatively studied the evolution of the two groups, by monitoring the variation of dependent variables (increase of muscle strength, joint angles and improvement of pain and joint stiffness) according to the independent variable (application of a kinesiotherapy program) in the study group, and the variation of dependent variables in the absence of the independent variable in the control group. 


\section{Results:}

The values of joint angles, muscle strength, Womac scale and Lequesne functional index increased in the patients of the study group after the 12 days of drug and kinesiotherapeutic treatment compared to day 1 before the beginning of treatment $(\mathrm{p}<0,05)$. ( Fig.nr.1,2,3,4).

After the 12 days of drug therapy, no changes in muscle strength, joint angles, pain and joint stiffness were found in patients of the control group.

\section{Conclusions:}

1. The kinesiotherapeutic treatment administered to patients of the study group (drug treatment and kinesiotherapy) contributed to the increase in joint angle values at the level of the coxofemoral joint.

2. After 12 kinesiotherapy sessions, we obtained an increase of muscle strength in patients of the study group compared to the control group.

3. The Womac test performed at the end of treatment (day 12) in the study group shows a decrease in pain intensity and a reduction in joint stiffness compared to day 1 before the beginning of treatment.

4. The Lequesne functional index applied at the end of the 12 days of treatment shows the fact that patients can easily walk longer distances and can perform daily activities more easily, which results in an increase of their quality of life.

\section{Bibliography:}

1. NICA AS. Compendiu de medicină fizică $i$ recuperare. Editura Universitară „CAROL DAVILA”, Bucure ti; 1998.

2. CERVINI C, FICHERA RC, GOUPILLE P, GRASSI W, TROTTA F. Teme alese de reumatologie. Editura Medicală Universitară „IULIU HA IEGANU" Cluj-Napoca; 2003.

3.

http://sanatateverde.blogspot.ro/2010/11/, accessed on 15 March 2013

4. IVAN G. Coxartroza. Editura Scrisul Românesc, Craiova; 1982. 
Mean joint angle values of the study group before and after treatment

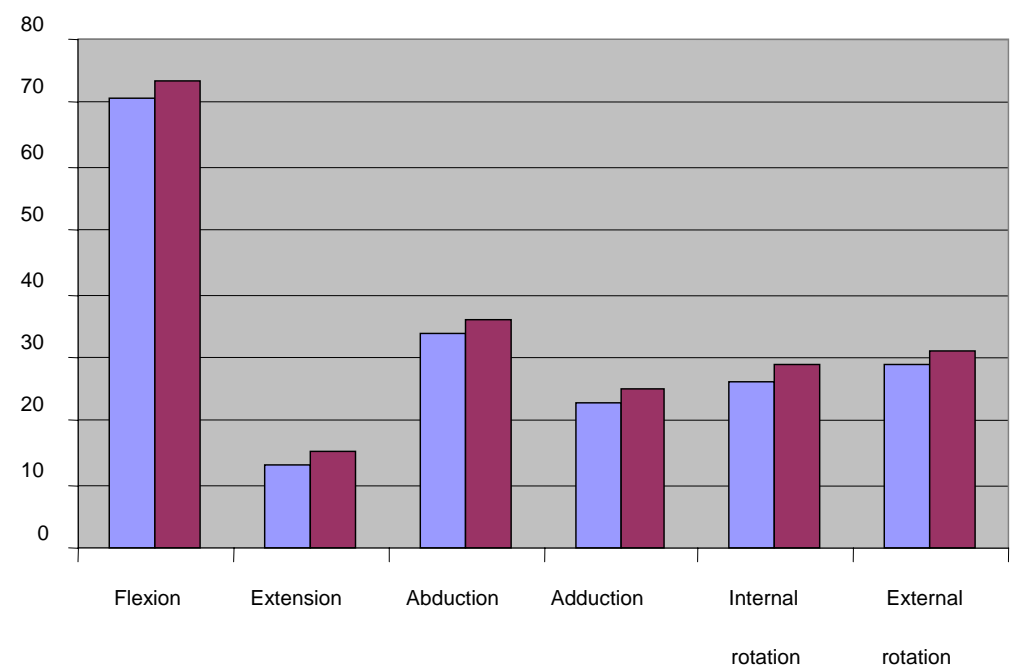

Values of the study group before treatment (day 1)

Values of the study group after

treatment (day 12)

Fig. 1. Mean joint angle values of the study group before and after treatment

Womac scale values for the assessment of pain in both groups after treatment

12

10

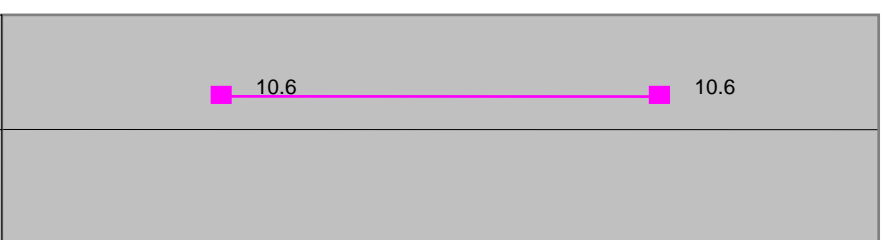

2

0

Day 1

Day 12

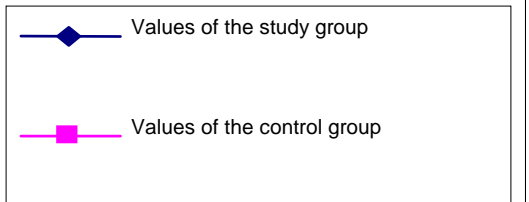

Fig. 2. Womac scale values for the assessment of pain in both groups after treatment 


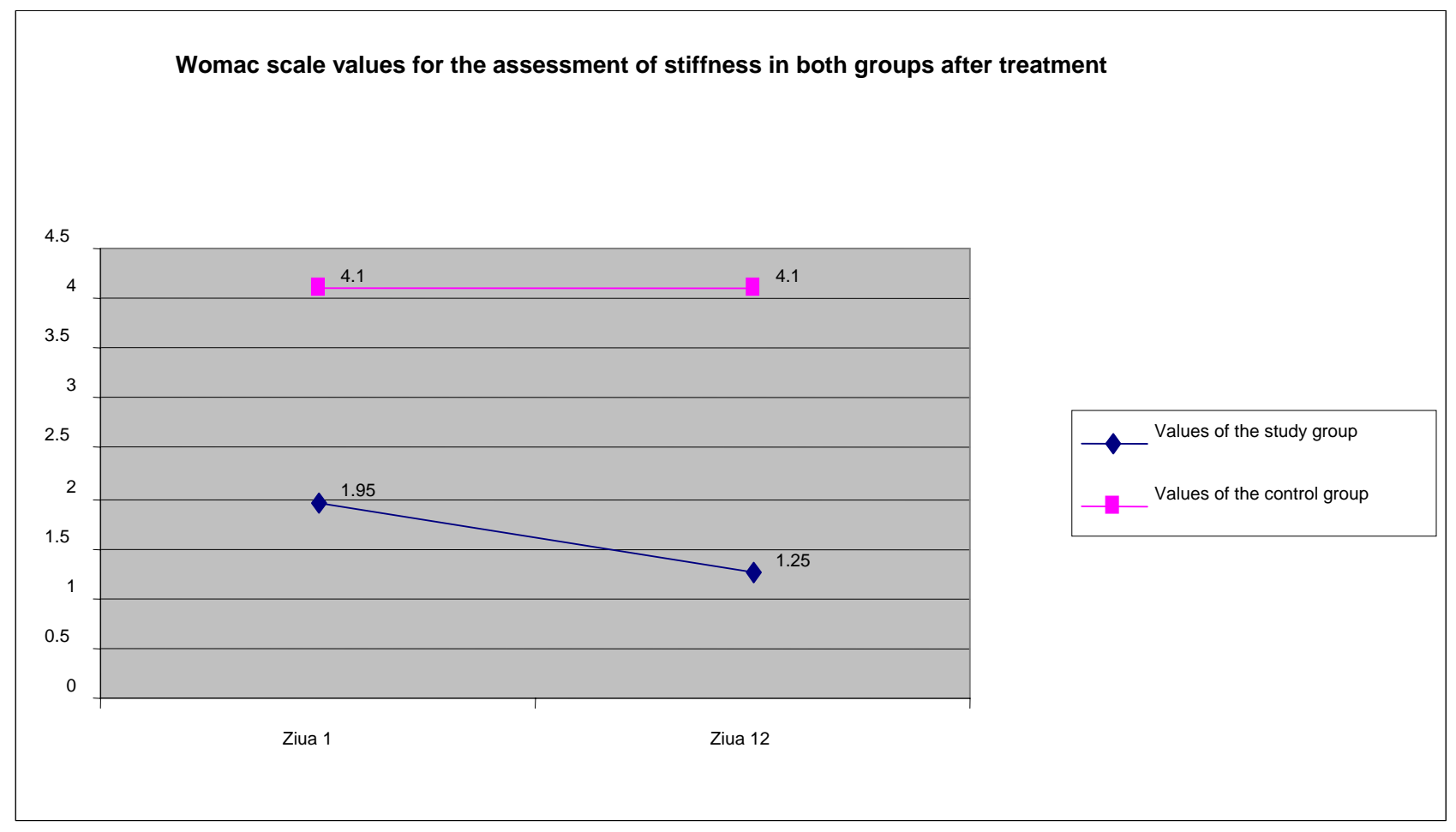

Fig. 3. Womac scale values for the assessment of stiffness in both groups after treatment

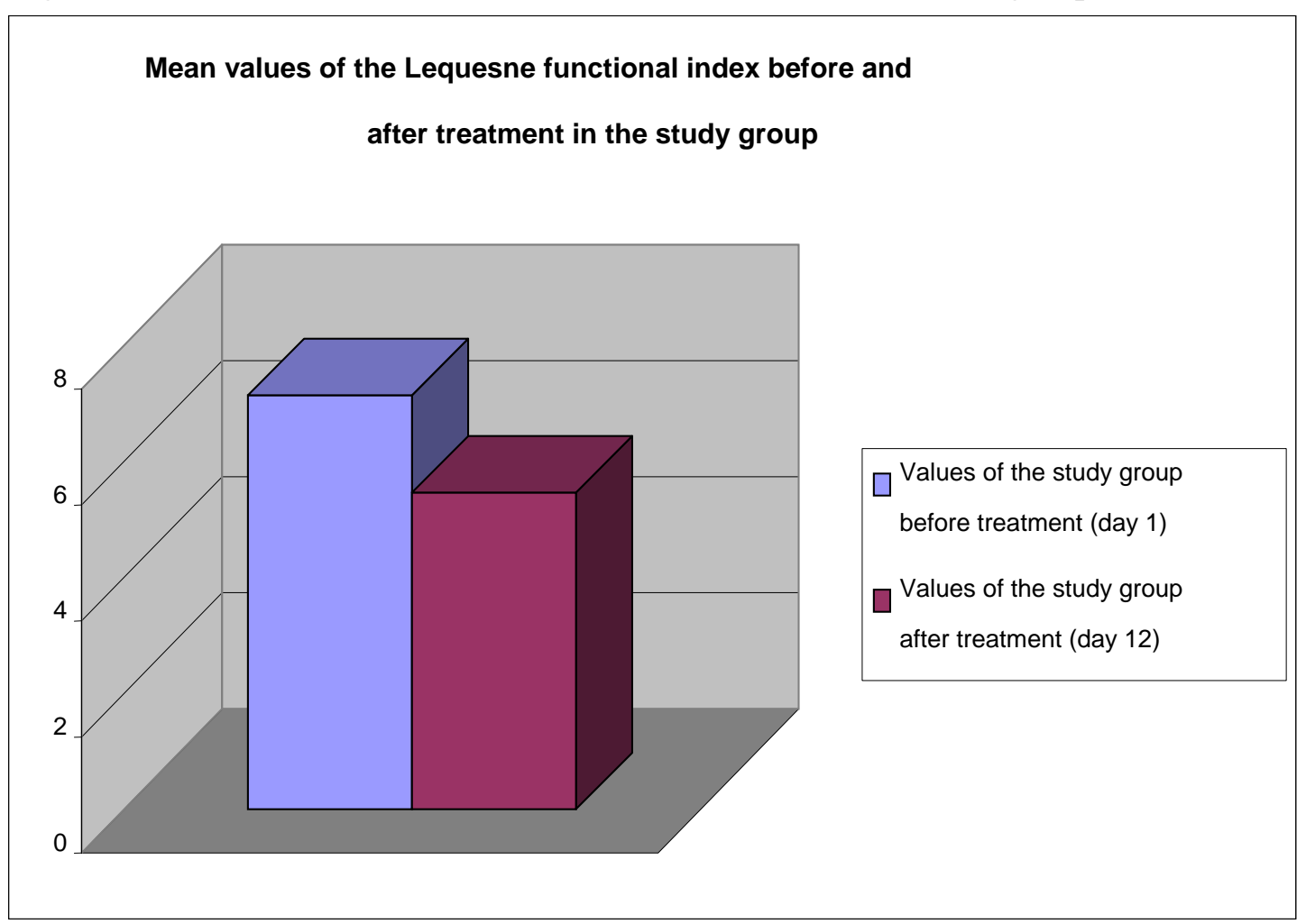

Fig. 4. Mean values of the Lequesne functional index before and after treatment in the study group 